\title{
Quantitative Variations in the Expression of the Mouse Serum Antigen Ss and Its Sex-Limited Allotype SIp
}

\author{
T. H. Hansen, ${ }^{1}$ T. N. Krasteff, ${ }^{1}$ and D. C. Shreffler ${ }^{1}$ \\ Received 22 Apr. 1974-Final 24 June 1974
}

A radial immunodiffusion assay for quantitation of the Ss and Slp serum antigens is described. Significant differences between the mean serum concentrations of Ss and Slp were found among various inbred strains. Some of these differences have been shown to be associated with the H-2 haplotype. The quantitative difference between Slp levels associated with the $\mathrm{H}-2^{\mathrm{a}}$ and $\mathrm{H}-2^{\mathrm{s}}$ haplotypes has been used as a marker for the $\mathrm{S}$ region in the analysis of certain $\mathrm{H}-2$ recombinant strains $[A . T H, B 10 . S(7 R), B 10 . S(9 R)$, and B10.BSVS]. Male mice of two strains with the $\mathrm{H}-2^{\mathrm{b}}$ haplotype have been shown to have significantly lower levels of SS compared to males of the other strains tested. Male mice of every strain examined were found to have significantly higher levels of Ss in their serum than females of the same strain. The molecular relationship and developmental patterns of the Ss and Slp antigens have also been investigated using the radial immunodiffusion assay.

KEY WORDS: mouse inbred strains; radial immunodiffusion; histocompatibility-2 locus $(H-2)$; serum antigens Ss, Slp.

\section{INTRODUCTION}

The mouse serum protein $\mathrm{Ss}$ is a serologically detected serum globulin (Shreffler and Owen, 1963). Heteroimmune antisera on Ouchterlony double diffusion revealed gross differences in the Ss levels of various inbred strains. In strain crosses, these differences appeared to be under the control of a

This research was supported by U.S.P.H.S. Program Project Grant GM 15419, U.S.P.H.S. Training Grant 2T01-GM-00071, and U.S.P.H.S. Research Career Development Award K3-HL-24980.

${ }^{1}$ Department of Human Genetics, University of Michigan, Ann Arbor, Michigan. 
single ( $S s$ ) gene. Inbred strains having the $H-2^{k}$ haplotype have low levels of Ss (Ss-L), while mice with other haplotypes have higher levels (Ss-H). This was the first indication of an association between the major histocompatibility complex, $H-2$, and the Ss serum protein.

In a search for Ss polymorphism, a sex-limited allotype (Slp) was found in the serum from males of particular Ss-H strains (Passmore and Shreffler, 1970). An alloantiserum was produced which defines the presence (Slp-a) or absence (Slp-o) of this antigen. This difference is controlled by a gene identical or very closely linked to Ss. Preliminary evidence was presented suggesting that the Slp specificity represents a structural difference on Ssreactive molecules (Passmore and Shreffler, 1970). Females of an Slp-positive strain have no detectable Slp reactivity, but it can be induced in these females by testosterone (Passmore and Shreffler, 1971).

The quantitative and qualitative variants of Ss have contributed importantly to the genetic mapping of the $H-2$ complex. Recombinant $H-2$ haplotypes have unequivocally placed the $S s$ locus in the genetic region (now denoted the $S$ region; Klein et al., 1974) located between the $K$ and $D$ regions, which code for the major $\mathrm{H}-2$ transplantation antigens (Klein and Shreffler, 1971).

It has been reported that the $S$ region is also involved in the mouse complement system (Démant et al., 1973). In several B10-congenic recombinant lines, complement levels were shown to be correlated with Ss-Slp phenotype. The Ss-H, Slp-a strains were found to have higher levels of complement than those Ss-H, Slp-o or those Ss-L, Slp-o.

Therefore, the $S$ region of the $H-2$ complex must be responsible for at least three functions. First, it determines the grossly different serum levels of Ss protein (Ss-H v. Ss-L). Second, it controls the presence or absence of a sex-limited alloantigenic determinant (s) on Ss molecules. Third, it influences the level of total hemolytic complement. The precise interrelationships of these three functions are presently unclear.

In this report, an improved quantitative assay for the Ss and Slp antigens is described, which has been employed to define further variations in the Slp levels of Slp-positive strains and in the Ss levels of Ss-H strains. In addition, the developmental patterns and molecular relationships of the Ss and Slp antigens have been reexamined in a more quantitative manner. (Preliminary data based on a semiquantitative assay were reported previously; Shreffler and Passmore, 1971.)

\section{MATERIALS AND METHODS}

\section{Mice and Sera}

The recombinant strains $\mathrm{B} 10 . \mathrm{S}(7 \mathrm{R})$ and $\mathrm{B} 10 . \mathrm{S}(9 \mathrm{R})$ were provided by $\mathrm{Dr}$. 
J. H. Stimpfling, and the B10.BSVS strain was provided by Dr. Martin Dorf. B10.BSVS animals were from the third backcross of the $H-2$ haplotype of the BSVS strain onto the B10 background. These mice were therefore heterozygous at $H-2\left(H-2^{t 5} / H-2^{b}\right)$ and not completely congenic with the B10 strain. All of the other inbred strains of mice were from our colony at the University of Michigan. DBA/2J male and female sera from animals 7-8 months old were purchased from The Jackson Laboratory.

\section{Antisera}

Rabbit anti-Ss was prepared by immunizing with partially purified Ss from pooled mouse serum. An immunization schedule described by Shreffler and Owen (1963) was followed. The heteroimmune antiserum was absorbed with pooled Ss-L serum. A single pool of anti-Ss was used for all assays.

The alloantiserum, anti-Slp, was prepared according to a method described by Passmore and Shreffler (1970). A single pool of antiserum produced in C3H.Q recipients immunized with partially purified Slp from DBA/2J male serum was used for all Slp quantitations.

\section{Quantitative Assay}

Radial immunodiffusion plates were prepared by a method similar to that described by Fahey and McKelvey (1965). Antiserum $(0.16 \mathrm{ml}$ anti-Slp or $0.8 \mathrm{ml}$ anti-Ss) was combined at $56 \mathrm{C}$ with $14 \mathrm{ml}$ of $1 \%$ agarose in $0.1 \mathrm{M}$ sodium phosphate buffer, $p \mathbf{H} 8$. This antiserum-agar mixture was immediately poured on a $3 \frac{1}{4}$ - by 4 -inch glass slide. Thirty equally spaced wells (diameter $3 \mathrm{~mm}$ ) were punched in the gel and filled with sample precisely to the gel surface. These radial diffusion plates were kept in a humid box at $37 \mathrm{C}$ for $24 \mathrm{hr}$. Plates were washed in physiological saline for $24 \mathrm{hr}$, dried with bibulous paper, and stained with $0.5 \%$ amido black in methanol-glacial acetic acid $(9: 1)$. The precipitin ring diameters were measured with a comparator eyepiece (Finescale). One pool of DBA/2J male serum was used as a standard. Four serial dilutions of the standard were included on every slide. A standard line was determined by linear regression of the precipitin ring diameter squared on the concentration of standard. The Ss or Slp concentrations for test sera were then calculated from the standard line and expressed in standard units. One standard unit (Ss unit or Slp unit) is defined as the concentration in the undiluted DBA/2J male serum.

\section{Statistical Comparisons}

Although test results were found to be reproducible from day to day, statistical comparisons of test samples were done only on a single radial diffusion plate, 
not between plates. This eliminated the introduction of extraneous sources of error. For statistical comparisons, radial diffusion plates were set up such that every plate contained serum from two different groups of five to seven animals, with replicates of each serum. All sera were collected and tested on the same day. A two-sided Student $t$-test was used to compare the mean concentrations of Slp or Ss of the two groups.

\section{Column Chromatography}

Sephadex G200 filtration was carried out at room temperature in a 1.5- by 90-cm column, which was equilibrated with $0.1 \mathrm{M}$ sodium phosphate buffer, $p \mathrm{H} 8$. Samples of $5 \mathrm{ml}$ whole serum were passed through the column. Fractions of $0.5 \mathrm{ml}$ were collected in a Buchler refrigerated fraction collector. Optical density at $280 \mathrm{~m} \mu$ was monitored on an ISCO ultraviolet analyzer.

\section{RESULTS}

\section{Differences Associated with $\boldsymbol{H}-\mathbf{2}$ Haplotype and Sex}

A compilation of all animals tested is given by strain and sex in Table I. The mean concentrations plus or minus the standard error of the mean are given for Slp concentration in Slp units and Ss concentration in Ss units. The total number of animals tested to determine each mean is given in parentheses. Because of developmental changes in levels of the Ss and the Slp antigens (see below), only mice older than 13 weeks of age were tested.

Substantial differences are seen between the different strains and sexes listed in Table I. The statistical significance of some of these differences was tested on the radial immunodiffusion plates listed in Table II. Anti-Slp was incorporated into the agar of plates $1-5$ and anti-Ss into the plates 6-15. Mean Ss or Slp concentrations of the two groups indicated on each plate were compared. The $t$-values and probability values are given for each comparison.

Inbred strains having the same $H-2$ haplotype seemingly have $S$ regions of the same origin. To test whether there are any differences in Ss or Slp expression associated with different $H-2$ haplotypes, strains differing only at $H$-2 (i.e., $H$-2-congenic lines) were compared. To test whether non- $H-2$ genes affect Ss or Slp expression, strains sharing the same $H-2$ haplotype but differing in background were compared.

The mean Slp level in male mice of the $H-2^{a}$ haplotype was significantly higher than that of males of the $\mathrm{H}-2^{\mathrm{s}}$ haplotype. Significant differences were seen both in the congenic pair B10.A, B10.S (Table II, plate 2) and in A, A.SW (plate 4). The mean concentrations of B10.A males and B10.S males 
Table I. Ss and Slp Levels in Sera from Different Strains and Sexes

\begin{tabular}{|c|c|c|c|c|c|}
\hline Strain & Allele $^{a}$ & Ss units & (No.) & Slp units & (No.) \\
\hline $\mathrm{A}_{\circlearrowleft}^{*}$ & $S s^{d}$ & $0.97 \pm 0.04$ & (11) & $1.01 \pm 0.03$ & (18) \\
\hline $\mathrm{A}+$ 웅 & $S s^{\mathrm{d}}$ & $0.43 \pm 0.02$ & (10) & - & \\
\hline A.BY ${ }_{0}^{*}$ & $S s^{b}$ & $0.56 \pm 0.04$ & (5) & - & \\
\hline A.SW & $S s^{s}$ & $0.95 \pm 0.05$ & (5) & $0.35 \pm 0.06$ & (12) \\
\hline A.SW웅 & $S s^{s}$ & $0.60 \pm 0.08$ & (5) & - & \\
\hline $\mathrm{B} 10_{0}^{*}$ & $S s^{b}$ & $0.65 \pm 0.04$ & (10) & - & \\
\hline B10 & $S s^{b}$ & $0.47 \pm 0.04$ & (10) & - & \\
\hline B10.A & $S s^{d}$ & $0.90 \pm 0.05$ & (18) & $0.68 \pm 0.04$ & $(25)$ \\
\hline B10.A우 & $S s^{d}$ & $0.46 \pm 0.04$ & (12) & 0 & (5) \\
\hline B10.D20 & $S s^{d}$ & $0.87 \pm 0.07$ & (8) & $0.67 \pm 0.04$ & (8) \\
\hline B10. $\mathrm{M}_{0}^{*}$ & $S s^{f}$ & $0.91 \pm 0.03$ & (10) & - & \\
\hline B10.M우 & $S s^{f}$ & $0.48 \pm 0.03$ & (10) & - & \\
\hline$B 10 . S_{0}^{+}$ & $S s^{s}$ & $0.84 \pm 0.06$ & (6) & $0.18 \pm 0.03$ & (25) \\
\hline$(\mathrm{B} 10 \times \mathrm{B} 10 . \mathrm{D} 2){ }^{n}$ & $S s^{b} / S s^{d}$ & $0.62 \pm 0.07$ & (7) & $0.33 \pm 0.05$ & (7) \\
\hline B10.Ko & $S s^{k}$ & $0.06 \pm 0.01$ & (5) & 0 & (5) \\
\hline $\mathrm{C} 3 \mathrm{H}_{0}^{*}$ & $S s^{k}$ & $0.05 \pm 0.01$ & (5) & - & \\
\hline $\mathrm{C} 3 \mathrm{H}$ 우 & $S s^{k}$ & $0.04 \pm 0.01$ & (5) & - & \\
\hline
\end{tabular}

a According to new nomenclature. See Discussion section.

Table II. Statistical Comparisons of Ss and Slp Levels in Different Strains and Sexes

\begin{tabular}{|c|c|c|c|}
\hline $\begin{array}{l}\text { Plate } \\
\text { No. }\end{array}$ & Strains compared & $t(\mathrm{df})$ & Probability level \\
\hline \multicolumn{4}{|c|}{ Slp plates } \\
\hline 1 & $\mathrm{~B} 10 . \mathrm{A}_{0}^{-}$and $\mathrm{A}_{0}^{*}$ & $t(9)=5.30$ & $<0.01$ \\
\hline 2 & B10.Aô and B10.Sô & $t(9)=6.8$ & $<0.01$ \\
\hline 3 & 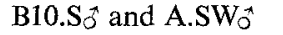 & $t(10)=3.12$ & $0.02-0.01$ \\
\hline 4 & A.SW ${ }_{0}^{\star}$ and $\mathrm{A}_{0}^{\star}$ & $t(10)=13.16$ & $<0.01$ \\
\hline 5 & B10.D2 ${ }^{\circ}$ and B10.A & $t(10)=1.78$ & $0.1-0.2$ (N.S.) \\
\hline \multicolumn{4}{|c|}{ Ss plates } \\
\hline 6 & $\mathrm{~B} 103$ and $\mathrm{B} 10$ ? & $t(8)=4.29$ & $<0.01$ \\
\hline 7 & $\mathrm{~B} 10 . \mathrm{A} \hat{o}$ and $\mathrm{B} 10 . \mathrm{A}$ 우 & $t(8)=3.85$ & $<0.01$ \\
\hline 8 & $\mathrm{~B} 10 . \mathrm{M}_{\odot}^{*}$ and $\mathrm{B} 10 . \mathrm{M}^{\circ}$ & $t(8)=4.62$ & $<0.01$ \\
\hline 9 & $A{ }^{-1}$ and $A$ ? & $t(11)=12.38$ & $<0.01$ \\
\hline 10 & A.SW ${ }^{*}$ and A.SW우 & $t(8)=4.83$ & $<0.01$ \\
\hline 11 & $\mathrm{~B} 10 \hat{\sigma}$ and B10.Mö & $t(8)=4.76$ & $<0.01$ \\
\hline 12 & $\mathrm{~B} 10 . \mathrm{A} \partial$ and B10.S & $t(10)=0.438$ & $0.8-0.6$ (N.S.) \\
\hline 13 & A.BY $\tilde{O}$ and $\mathrm{B} 10 \tilde{\sigma}$ & $t(8)=0.488$ & $0.8-0.6$ (N.S.) \\
\hline 14 & $\mathrm{~A}_{0} \overrightarrow{\text { and }} \mathrm{B} 10 . \mathrm{A} \sigma$ & $t(8)=0.0$ & 0.0 (N.S.) \\
\hline 15 & 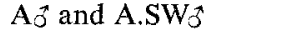 & $t(8)=0.31$ & $0.8-0.6$ (N.S.) \\
\hline
\end{tabular}


were $0.68 \mathrm{Slp}$ unit and $0.18 \mathrm{Slp}$ unit, respectively, while those in A males and A.SW males were $1.01 \mathrm{Slp}$ units and $0.35 \mathrm{Slp}$ unit, respectively (Table I).

Male mice of strain B10.A were found not to differ significantly in Slp levels from B10.D2 male mice (Table II, plate 5). This is expected since $H-2^{a}$ very probably derived its $S$ region from $H-2^{d}$ ( $c f$. Klein and Shreffler, 1971).

In contrast to the A background, the B10 background diminishes Slp expression. This reduction was found to be significant both in the B10.A male and A male combination (Table II, plate 1) and in B10.S male and A.SW male combination (plate 3 ).

The strains which showed significant differences in Slp level nevertheless failed to differ in Ss level (Table II, plates 12, 14, and 15). Even though B10.S and A.SW males have lower Slp levels than B10.A and A, respectively, all of them have similar Ss levels (Tables I and II). Therefore, no $\mathrm{H}-2-$ associated or background differences in Ss levels were seen in these strains. Since lower levels of Ss are not concomitant with lower levels of Slp, these two antigens are apparently regulated independently. Further evidence for the independent regulation of the two antigens was seen in the developmental patterns of Slp-positive strains.

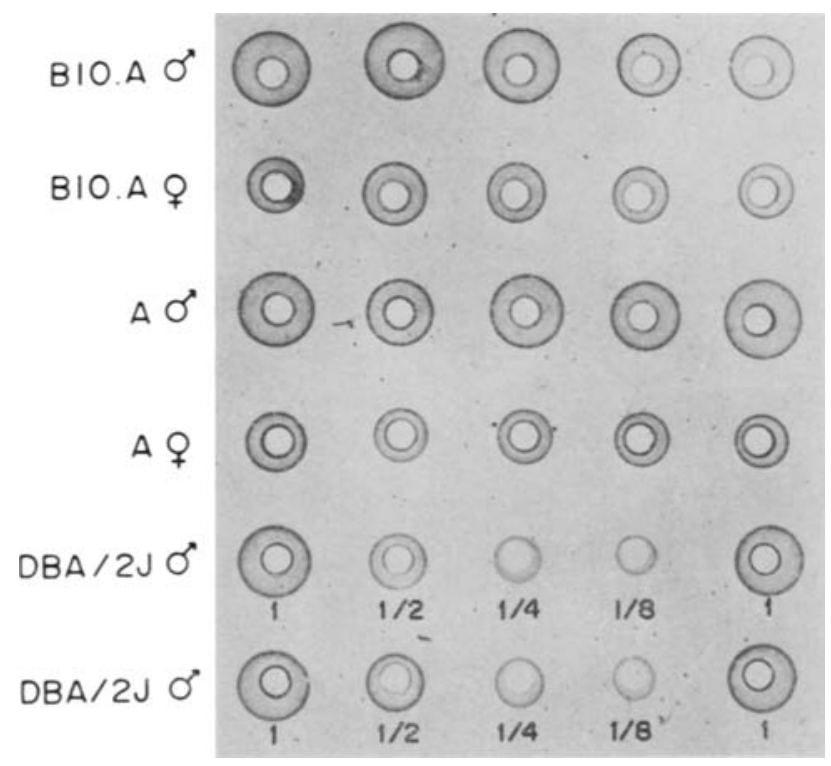

Fig. 1. Radial immunodiffusion plate showing differences in Ss levels between male and female sera of strains B10.A and A. The top four rows of the plate were filled with sera from five different adult mice of the indicated strain and sex. Serial dilutions of standard were added to the bottom two rows. 
Interstrain differences in Ss levels were seen. Males of two strains having the $H-2^{b}$ haplotype were found to have significantly lower levels of Ss than the other strains examined (Table II, plates 11 and 13). A.BY males and B10 males had mean concentrations of 0.56 and 0.65 Ss unit, respectively. Strains having the $H-2^{b}$ haplotype are Slp negative, while the $H-2^{a}$ and $H-2^{s}$ strains considered above are Slp positive. One possible explanation was that the reduction in Ss level was correlated with the Slp-negative phenotype. This, however, seems not to be the case. Strain B10.M male mice, which are Slp negative, had a mean Ss level (0.91 Ss unit) similar to that of the Slp-positive strains that were tested (Table I). The B10.M males were found to have significantly higher Ss levels than B10 males (Table II, plate 11). The lower level of Ss appeared to be dominant in the $(\mathrm{B} 10 \times \mathrm{B} 10 . \mathrm{D} 2) \mathrm{F}_{1}$ hybrid males (Table I). However, these hybrids were found to have a mean Slp level equal to about half that of B10.D2 males.

Significant differences in the Ss levels of males and females were seen in every strain tested (Table II, plates 6-10). This male-female disparity was previously shown to be hormone dependent (Shreffler and Passmore, 1971). A radial diffusion plate showing the contrast between the Ss levels in males and females is shown in Fig. 1.

Two strains that have the $H-2^{k}$ haplotype are included in Table I. As indicated earlier (Shreffler and Owen, 1963), these animals have approximately twenty-fold lower levels of Ss.

Table III. Determination of Slp Levels in Two Intra-H-2 Recombinants

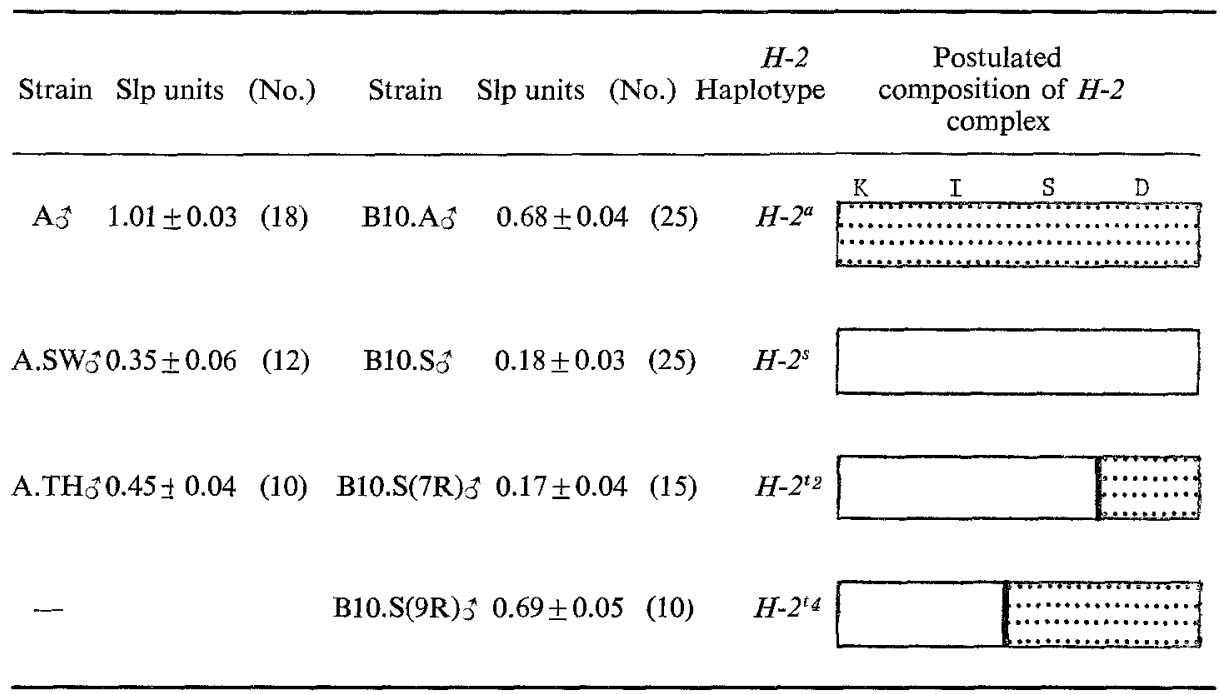




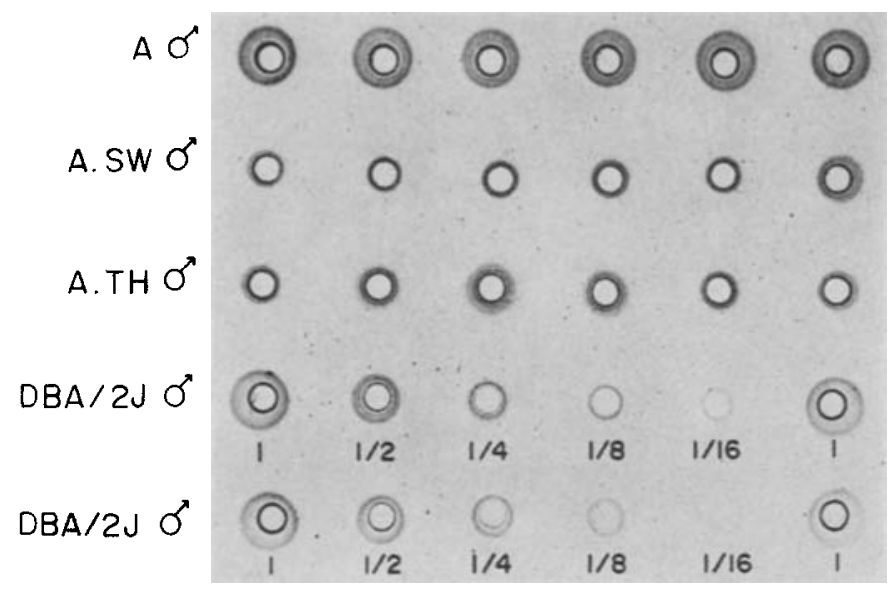

Fig. 2. Radial immunodiffusion plate comparing Slp levels in sera of males of strains A, A.SW, and A.TH. Note similarity of levels in A.SW and A.TH. Serum samples from six different adult male mice of the indicated strain were added to the top three rows. The bottom two rows were filled with serial dilutions of standard.

\section{Typing of $\boldsymbol{H - 2}$ Recombinant Strains}

These quantitative differences in Slp level between $H-2$ haplotypes have provided additional markers for identifying the origin of the $S$ region in certain $H-2$ recombinant strains. Three recombinant strains, which arose from crossovers between $H-2^{a}$ and $H-2^{s}$, are listed in Table III. Also included in the table are the parental strains from which the recombinants were derived. In order to locate the crossover position, the recombinant strains were typed to determine the origin of each of their $H-2$ regions $(K, I, S, D)$. Since there is a quantitative difference in Slp level between the $H-2^{a}$ and $H-2^{s}$ types, these recombinants could be Slp-typed and the origin of their $S$ regions unambiguously determined.

A radial diffusion plate showing the level of Slp in A.TH male serum is shown in Fig. 2. Included on this plate are serum samples from $H-2^{a}$ controls (A males) and $H-2^{s}$ controls (A.SW males). A.TH males and A.SW males were found to have similar levels of Slp ( 0.45 and $0.35 \mathrm{Slp}$ unit, respectively), both of which are considerably different from that found in A males (1.01 Slp units). B10.S(7R) derived its recombinant haplotype from the same original crossover as A.TH. Therefore, as expected, B10.S(7R) males and B10.S males were found to have similar mean concentrations of serum Slp ( 0.17 and $0.18 \mathrm{Slp}$ unit, respectively), both of which are considerably different from that found in B10.A males (0.68 Slp unit). 
B10.S(9R) male mice were found to have a mean Slp concentration $(0.69$ Slp unit) like that of the $H-2^{a}$ control, B10.A males. The typing of these recombinants for the other $H-2$ regions besides $S$ has been published elsewhere ( $c f$. Shreffler and David, 1974).

B10.BSVS carries another $H-2$ haplotype which probably arose from a crossover between the $H-2^{a}$ and $H-2^{s}$ haplotypes. Evidence from a limited number of animals of the partially congenic B10.BSVS strain indicates that the $S$ region of this strain was derived from the $H-2^{a}$ haplotype. However, because the line is not yet entirely congenic and background genes are known to affect Slp levels, a definitive conclusion is not yet possible.

Use of Slp level as a marker for the $S$-region of course assumes that the genetic determinant responsible for these dissimilar Slp levels lies within the $S$-region. We have no present reason to question that assumption.

\section{Developmental Patterns of Ss and SIp}

Mice of various ages from 3 to 18 weeks were bled and tested for Slp and Ss levels. The B10.A developmental pattern is shown in Fig. 3. Both Ss and Slp levels increase in males in the period 3-11 weeks of age. However, the mean Ss level rises earlier and reaches a plateau sooner than the mean Slp level. Female Ss levels remain constant throughout maturation. Strains B10.D2 and $\mathrm{C} 3 \mathrm{H} . \mathrm{OH}$ gave developmental patterns very similar to that shown in Fig. 3.

\section{Molecular Relationship of Ss and Slp}

To further elucidate the relationship between the Ss and Slp antigenic sites, whole sera from DBA/2J males and DBA/2J females were separated on a Sephadex G200 column. Fractions were assayed for $\mathrm{OD}_{280}$, Ss reactivity, and Slp reactivity. The results are shown in Fig. 4. Two peaks of Ss reactivity

Fig. 3. Changes in serum Ss and Slp levels during postnatal development in strain B10.A. O-O, Ss level in males; $0_{-}-O$, Slp level in males; ..., , Ss level in females.

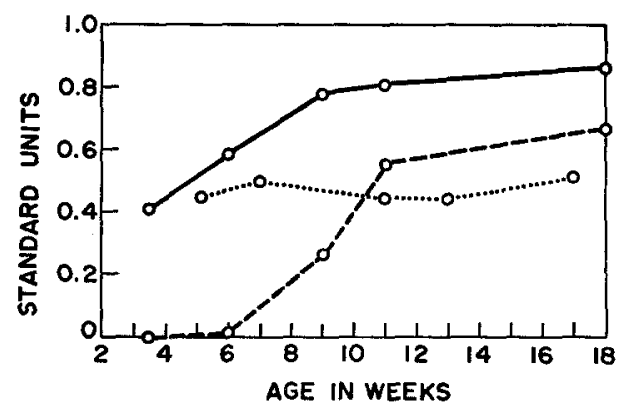



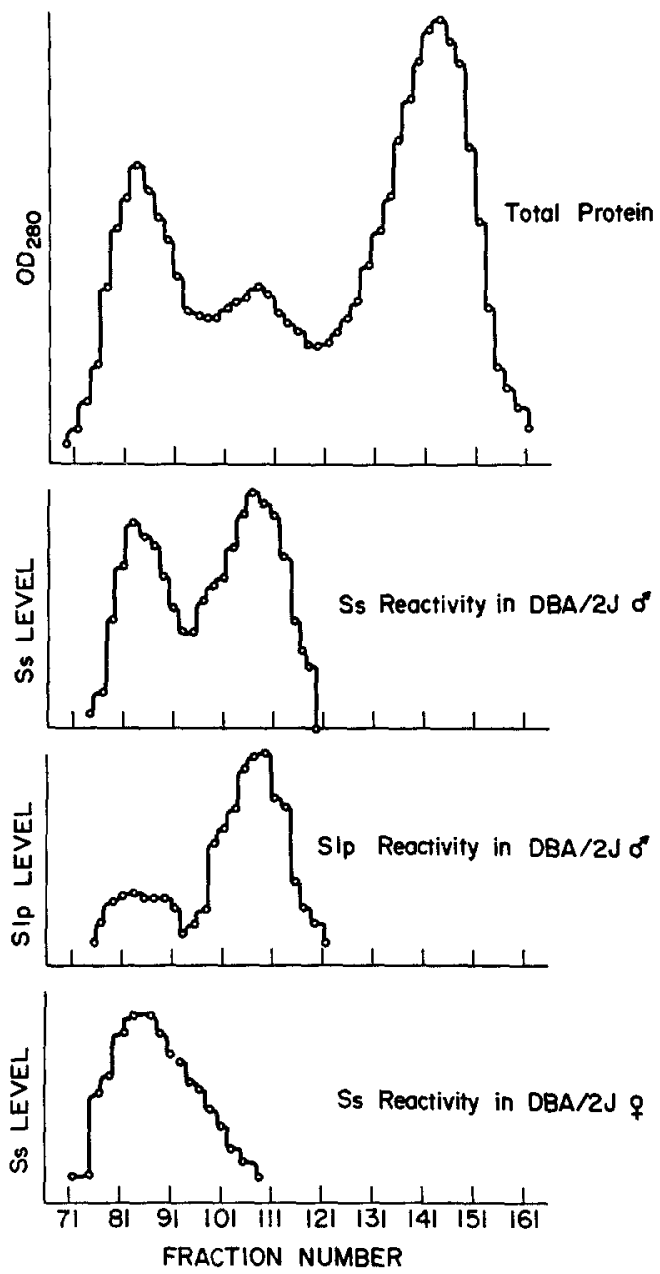

Fig. 4. Sephadex G200 profiles of Ss and Slp antigens in DBA/2J male and female sera.

were seen in the male serum, but only the higher molecular weight peak was seen in female serum. Although the predominant amount of Slp reactivity in male serum coincided with the second Ss peak, every fraction having Ss reactivity also had Slp reactivity and vice versa.

Reciprocal absorptions of DBA/2J male serum were done with anti-Ss and anti-Slp, according to a procedure previously described (Passmore and Shreffler, 1970). Figure 5 shows the results of preabsorption with different concentrations of anti-Ss, followed by testing with anti-Ss and anti-Slp. 
Fig. 5. Effect of preabsorption of $\mathrm{DBA} / 2 \mathrm{~J}$ male serum with anti-Ss on Ss and Slp activities. Anti-Ss was mixed with the serum at varying ratios and incubated $16 \mathrm{hr}$ at $4 \mathrm{C}$. The mixtures were centrifuged and the supernatants assayed for remaining Ss and Slp activity. Normal rabbit replaced anti-Ss in control mixtures. $0 \cdots$, Slp activity of antiSs mixtures; $0---0$, Ss activity of antiSs mixtures; $0 \cdots 0$, Slp activity of control mixtures; O-O; Ss activity of control mixtures.

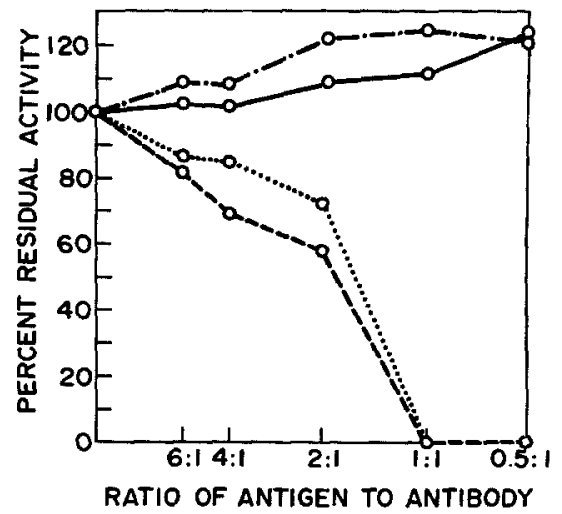

The removal of Slp reactivity was concomitant with the removal of Ss reactivity. In the control, normal rabbit serum was added instead of anti-Ss. The curves in Fig. 5 clearly show that, on addition of increasing amounts of anti-Ss, Ss and Slp levels decline uniformly and in parallel, indicating that all molecules with Slp determinants also have Ss determinants.

Figure 6 shows the results of preabsorption with different concentrations of anti-Slp, followed by testing with anti-Slp and anti-Ss. (The anti-Slp

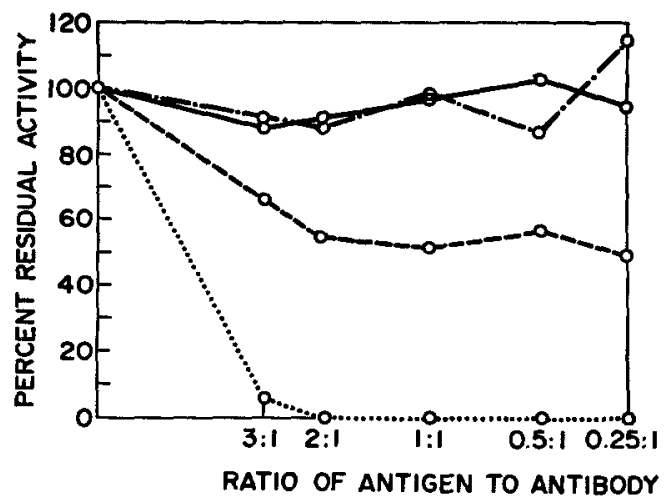

Fig. 6. Effect of preabsorption of DBA/2J male serum with anti-Slp on Ss and Slp activities. AntiSlp produced in an Ss-L strain was mixed with the serum at varying ratios and incubated $16 \mathrm{hr}$ at $4 \mathrm{C}$. The mixtures were centrifuged and the supernatants assayed for remaining Ss and Slp activity. Normal Ss-L serum replaced anti-Slp in control mixtures. $0 \cdots$, Slp activity in anti-Slp mixture; $0_{-}-0_{\text {, }}$ Ss activity in anti-Slp mixtures; $\mathrm{O}_{-} \mathrm{O}$, Slp activity in control mixtures; $0-. .$, , Ss activity in control mixtures. 
used as the absorbant for this experiment was from an Ss-L strain, therefore adding minimal amounts of Ss to the mixture.) At a 2:1 ratio of antigen to absorbant, all of the Slp reactivity was removed, whereas the Ss reactivity was not completely removed either at this point or with addition of further anti-Slp. In the control, DBA/2J male serum was mixed with normal Ss-L serum. The difference in the Ss reactivity curves in Fig. 6 indicates that in DBA/2J male serum about half of the Ss-reactive molecules have no Slp determinants.

\section{DISCUSSION}

Significant differences have been demonstrated above in the levels of expression of the Ss and Slp antigens in various inbred strains. Some of these disparities can be attributed to non- $H-2$ genes. For example, the B 10 background, in contrast to the A background, reduces Slp expression. Other quantitative differences in Ss and Slp antigen levels must be $H$-2-associated. For example, lower levels of Ss antigen are associated with the $H-2^{b}$ haplotype than with $H-2^{f}$, and lower levels of Slp are associated with $H-2^{s}$ than $H-2^{a}$. The H-2associated differences in the levels of Ss and Slp seemingly reflect genetic differences within the $S$ region of the $H-2$ complex. This suggests that the $S$ regions of different Ss-H or Slp-a strains are not genetically identical; i.e., there are a number of different alleles, associated with different $\mathrm{H}-2$ haplotypes, which determine various discrete levels of Ss or Slp antigen in serum. Since it has not been determined whether the Ss and Slp traits are controlled by the same gene or by two different genes, it is perhaps misleading to designate separate $S s$ and $S l p$ loci and alleles. We therefore propose that for the sake of simplicity of notation the gene(s) controlling Ss or Slp variation should be denoted simply by the symbol $S s$, with an allelic superscript denoting the $H-2$ haplotype of origin. For example, the $S s$ allele associated with the $S$ region of the $H-2^{b}$ haplotype should hereafter be designated as $S s^{b}$, the allele associated with the $H-2^{d}$ haplotype as $S s^{d}$, and so forth. This nomenclature system is consistent with the nomenclature used for the rest of the $\mathrm{H}-2$ gene complex (Klein et al., 1974). The Ss alleles listed in Table I have been designated according to this convention.

There may be other regions of the $H-2$ complex which influence Ss and Slp expression. Ivanyi et al. (1972a,b) found that male B10 mice, as compared to their congenic partner B10.A, have lower levels of plasma testosterone, smaller testicles, and larger vesicular glands. The genetic determinant responsible for these hormone effects (Hom-l) has not been precisely mapped within the $H-2$ complex. Since Ss levels are known to be hormone dependent (Shreffler and Passmore, 1971), the lower levels of testosterone might be either the cause or the effect of the lower levels of Ss found in $H-2^{b}$ male mice. 
Both the lower level of plasma testosterone (Ivanyi et al., 1972b) and the lower level of Ss were found to be dominant in the $F_{1}$ hybrid.

It has been tacitly assumed in these studies that interstrain differences in Ss and Slp expression reflect unequal numbers of reactive molecules. If this is the case, there must be genetic variations (within the $S$ region) of a regulatory gene (or genes). However, these interstrain differences could also result from differences in structural genes leading to subtle structural differences in the Ss protein. For example, alterations in the number, the affinity, or the accessibility of the Ss or Slp antigenic sites might give the same dissimilarity in Ss and Slp expression without affecting the number of reactive molecules. So the paramount question is whether the $S$ region of the $H-2$ complex contains regulatory or structural genes or both. Further biochemical and genetic analyses of the Ss and Slp antigens will be required to answer this question.

\section{ACKNOWLEDGMENT}

We are grateful to Dr. Peter Smouse for his advice on the statistical analysis of the data.

\section{REFERENCES}

Démant, P., Capkoká, J., Hinzová, E., and Vorácová, B. (1973). The role of the histocompatibility-2-linked Ss-slp region in the control of mouse complement. Proc. Natl. Acad. Sci. 70:863.

Fahey, J. L., and McKelvey, E. M. (1965). Quantitative determination of serum immunoglobulins in antibody-agar plates. J. Immunol. 94:84.

Ivanyi, P., Gregorová, S., and Micková, M. (1972a). Genetic differences in thymus, lymph node, testes and vesicular gland weights among inbred mouse strains: Association with the major histocompatibility $(H-2)$ system. Folia Biol. 18:81.

Ivanyi, P., Hampl, R., Starka, L., and Micková, M. (1972b). Genetic association between a histocompatibility gene $(H-2)$ and hormone metabolism in mice. Nature 238nb:280.

Klein, J., and Shreffler, D. C. (1971). The $H-2$ model for the major histocompatibility system. Transplant. Rev. 6:3.

Klein, J., Bach, F., Festenstein, H., McDevitt, H. O., Shreffler, D. C., Snell, G. D., and Stimpfling, J. H. (1974). Genetic nomenclature for the $H-2$ complex of the mouse. Immunogenetics 1:184.

Passmore, H. C., and Shreffler, D. C. (1970). A sex-limited serum protein variant in the mouse: Inheritance and association with the $H-2$ region. Biochem. Genet. 4:351.

Passmore, H. C., and Shreffler, D. C. (1971). A sex-limited serum protein variant in the mouse: Hormonal control of phenotypic expression. Biochem. Genet. 5:201.

Shreffler, D. C., and David, C. S. (1974). The H-2 major histocompatibility complex and the $I$ immune response region: Genetic variation, function and organization. Advan. Immunol. 20:125.

Shreffler, D. C., and Owen, R. D. (1963). A serologically detected variant in mouse serum: Inheritance and association with the histocompatibility-2 locus. Genetics 48:9.

Shreffler, D. C., and Passmore, H. C. (1971). Genetics of the $H-2$ associated Ss-Slp trait. In Lengerová, A., and Vojtisková, M. (eds.), Immunogenetics of the H-2 System, Karger, Basel, p. 85. 